\title{
ANÁLISIS DE LA AUTENTICIDAD DE UNA TAREA DESDE LA PERSPECTIVA DE UN RESOLUTOR
}

ANALYSIS OF THE AUTHENTICITY OF A TASK FROM THE PERSPECTIVE OF A SOLVER

\author{
María Fernanda Pichardo Zamora \\ Benemérita Universidad Autónoma de Puebla. México \\ fernanda.pichardo@alumno.buap.mx \\ Estela de Lourdes Juárez Ruiz \\ Benemérita Universidad Autónoma de Puebla. México \\ estela.juarez@correo.buap.mx
}

\begin{abstract}
Resumen
En este artículo se presenta un análisis de autenticidad de una tarea de geometría analítica desde la perspectiva de un alumno de educación media superior; se realizó por medio de una entrevista clínica. Dicho análisis tiene como objetivo identificar cuáles son los aspectos de autenticidad que no cumple la tarea desde la visión del resolutor y su relación con las dificultades que se le presentaron al resolver la tarea. Los resultados indican que el estudiante logró identificar que los aspectos Pregunta y Especificidad de los datos no se cumplen y el análisis de su producción escrita permitió observar dificultades al resolver la tarea en dos de las preguntas. Se concluye que la falta de autenticidad de las tareas puede incidir directamente en dificultades al momento de resolverlas.
\end{abstract}

Palabras clave: autenticidad, problemas verbales, matemáticas

\begin{abstract}
This article presents an analysis of the authenticity of an analytic geometry task from the perspective of a high school student; it was done through a clinical interview. This analysis aims to identify which are the authenticity aspects that the task does not fulfill from the solver's point of view; also, to identify the relationship with the difficulties that arose when solving the task. The results indicate that the student was able to identify that the Question and Specificity aspects of the data are the ones that were not fulfilled, and the analysis of his written production allowed to observe the difficulties when solving the task in two of the questions. It is concluded that the lack of authenticity of the task can directly affect difficulties when solving it.
\end{abstract}

Keywords: authenticity, word problems, mathematics

Investigación e Innovación en Matemática Educativa (2020) Volumen 5

Recibido: Agosto 26, 2020. Aceptado: Noviembre 17, 2020. Publicado: Diciembre 14, 2020. 


\section{INTRODUCCIÓN}

En este trabajo se realiza el análisis de autenticidad de una tarea de geometría analítica desde la perspectiva de un estudiante de nivel medio superior, la cual fue seleccionada de un libro de texto. La palabra tarea tiene diferentes significados. Para Leont'ev (1975) significa una operación realizada dentro de ciertas restricciones y condiciones; para Mason y Johnston-Wilder (2006) es lo que se pide a los estudiantes; para Becker y Shimada (1997) son materiales o entornos diseñados destinados a promover una actividad matemática compleja. En este estudio, el concepto de tarea que más se ajusta a la actividad seleccionada es el que enuncian Watson et al. (2014):

Usamos 'tarea' para referirnos a una gama más amplia de 'cosas que hacer' e incluimos ejercicios repetitivos, construcción de objetos, ejemplificación de definiciones, resolución de problemas de una o varias etapas, decidir entre dos posibilidades, o realización de un experimento o investigación.

Cualquier cosa que un profesor utiliza para demostrar matemáticas, para realizar una actividad interactiva con los estudiantes o para pedirles que hagan algo. La tarea también puede ser cualquier cosa que los estudiantes decidan hacer por sí mismos en una situación particular (p. 9-10).

Utilizaremos indistintamente problema y tarea. Es preciso aclarar que la palabra problema se utiliza para hablar de una situación de la cual se busca un resultado. En el ámbito escolar, Labarrere (1996) se refiere a él como dirigido a cumplir con los objetivos y contenidos de la enseñanza matemática. Para Codina y Rivera (2001), un problema es una tarea para la cual el individuo o grupo que se enfrenta a ella quiere o necesita encontrar una solución; no hay un procedimiento fácilmente accesible que garantice o determine completamente la solución, y el individuo o grupo debe realizar intentos para encontrar la solución. Como se puede observar en esta última definición, la resolución de un problema es un caso particular de una tarea.

Acerca de los problemas auténticos en la enseñanza de la ciencia, Jiménez (1998) se refiere a un problema auténtico como aquel que implica una situación que tiene la misma complejidad de la realidad y contextualizada en la vida cotidiana. Según Duschl y Gitomer (1996) dicha autenticidad tiene dos componentes: el contexto del problema y su relevancia para los estudiantes.

En este trabajo nos referimos a la autenticidad de una tarea matemática como la concordancia entre una tarea escolar que incluya una descripción de una situación real y la situación real. El

Investigación e Innovación en Matemática Educativa (2020) Volumen 5

https://doi.org/10.46618/iime.85 
término "tareas auténticas" hace referencia a tareas escolares contextualizadas que emulan una situación de la vida real (Palm, 2008).

En este estudio se realizó un análisis mediante una entrevista clínica para identificar la autenticidad de una tarea. Se utilizaron cinco aspectos establecidos por Palm y Nyström (2009) como resultado y síntesis de un análisis que estos autores realizaron a los siete aspectos previamente establecidos por Palm (2006).

Es de nuestro interés analizar la autenticidad de la tarea propuesta ya que algunos estudios muestran que pequeñas modificaciones en las tareas, pueden favorecer su resolución influyendo en el comportamiento de los estudiantes. En particular, Palm (2008) demostró que un aumento en la autenticidad de la tarea, aumenta la tendencia de los estudiantes a utilizar su conocimiento del mundo real de manera efectiva en los problemas planteados y de esta manera tener un mayor éxito en la resolución.

Existen diversos trabajos acerca de la importancia de la teoría de situaciones auténticas. Por ejemplo, en Martínez (2019) se analizan las estrategias utilizadas por un grupo de estudiantes de una localidad en la implementación de una tarea matemática auténtica diseñada a partir de un problema geométrico detectado en su entorno, la cubicación de la madera. Al desarrollar la tarea se logró que los estudiantes reconocieran y valoraran la utilidad de las matemáticas fuera del aula, así como el reconocimiento del potencial de los recursos matemáticos y no matemáticos con los que cuentan para resolver problemas de su comunidad.

Otro caso es el que se muestra en De León (2020), en donde se realiza un análisis de libros de texto de matemáticas de educación secundaria de México y Colombia en el tema de temperaturas. Como resultado se muestra que los problemas planteados en libros mexicanos, en su mayoría, cumplen a lo más con un aspecto de autenticidad de la teoría de Palm, y que los que se proponen en los libros colombianos no cumplen con ningún aspecto. Esto deriva en que los alumnos no utilicen su conocimiento del mundo real al resolverlos, además de que dejan a un lado el contexto de los problemas y sólo se enfocan en los números.

Investigación e Innovación en Matemática Educativa (2020) Volumen 5 https://doi.org/10.46618/iime.85 
En este trabajo se busca responder la pregunta: ¿Cómo percibe un estudiante de bachillerato los aspectos de autenticidad de una tarea y cómo estos se relacionan con dificultades en sus procesos de resolución?

El objetivo de esta investigación es identificar cuáles son los aspectos de una tarea propuesta que percibe como auténticos un estudiante de bachillerato, y cómo se relacionan con dificultades que presenta en su resolución.

La investigación está enfocada en las dificultades que puede presentar un resolutor derivado de la falta de autenticidad de una tarea matemática propuesta, sin profundizar en otras dificultades en los procesos cognitivos que le impidan al resolutor llegar a la respuesta, tales como visualización, idealización o comprensión lectora.

\section{MARCO TEÓRICO}

En los libros de texto se plantean tareas que simulan ser de la vida real. Como ejemplo se toma el caso del libro de Ruiz (2014) el cual menciona que en él se encontrarán ejercicios que ayudarán al estudiante a solucionar problemas que se le presenten en su vida cotidiana; sin embargo, existen diversos estudios que aseveran lo contrario. Tal es el caso de la investigación de Guerrero, Carrillo y Contreras (2014) quienes afirman que en los libros de texto de matemáticas predomina el contexto puramente matemático seguido por el de la vida real con la presencia de datos no realistas y contextos sin conexión. En los problemas verbales que se asocian a contextos de la vida real, el realismo solo es parcial pues los datos son inventados.

Una consecuencia de la falta de autenticidad en las tareas podría ser el bajo desempeño en la materia de matemáticas, por lo que es de suma importancia identificar qué tareas en los libros de texto son auténticas y en caso de no serlo identificar cómo interviene este hecho en los procesos de resolución de los estudiantes. La dificultad puede tener su origen en la falta de autenticidad en el planteamiento de la tarea, pero el docente puede confundirla con otras razones del mal desempeño de los estudiantes en la resolución de problemas, tales como: falta de conocimientos, falta de comprensión de conceptos o errores en los procedimientos matemáticos.

Investigación e Innovación en Matemática Educativa (2020) Volumen 5

https://doi.org/10.46618/iime.85 
La mayoría de los problemas que aparecen en los libros de texto de matemáticas que simulan ser contextualizados en la vida real, en realidad son problemas puramente matemáticos escritos en un lenguaje cotidiano (Blum y Niss, 2014).

En Palm (2006) se propone un marco para analizar la concordancia entre problemas verbales y situaciones del mundo real, más allá del aula de matemáticas. Esto surgió debido a que muchos de los problemas en el aula no son simulaciones reales de situaciones extra-escolares, sino meras tareas vestidas con un contexto figurativo que retoma un contexto de fuera de la escuela. La falta de realismo podría tener un impacto negativo en la resolución de la tarea.

La Teoría de Situaciones Auténticas propuesta por Palm (2006) es una teoría que puede ser útil para analizar problemas de tipo textual que emulan situaciones reales, de tal forma que los estudiantes se familiaricen con matemáticas útiles en situaciones fuera de la escuela y en la práctica de resolución de problemas que requieran circunstancias que se consideran como situaciones en la vida diaria.

Aunque en Palm (2006) se plantean siete aspectos de autenticidad, algunos con diversos sub-aspectos, en este trabajo analizaremos los cinco aspectos que se mencionan en Palm y Nyström (2009) como fundamentales en la autenticidad de una tarea. Se retoma la forma en que aparecen en el análisis que hacen Vicente y Manchado (2017); se describen a continuación:

1) Evento: Debe ser una situación que es probable que el alumno pueda vivir fuera de la escuela.

2) Pregunta: Podría ser formulada de manera habitual para el evento descrito en la tarea. La respuesta a las preguntas tiene un valor práctico o es interesante para otros no interesados en las matemáticas.

3) Existencia de datos: Los datos proporcionados en la tarea coinciden con los datos accesibles en la vida real.

4) Propósito en el contexto figurativo: Se menciona explícitamente en la tarea el propósito que se persigue con su resolución, que coincide con el que cabría plantearse en la vida real.

Investigación e Innovación en Matemática Educativa (2020) Volumen 5 https://doi.org/10.46618/iime.85 
5) Especificidad de los datos: Los personajes en la tarea tienen nombre propio, los objetos están definidos y los lugares son específicos, o bien el problema está formulado en primera o segunda persona.

Se considerará una tarea auténtica si cumple con estos cinco aspectos.

Con la finalidad de explicitar a qué se refieren estos aspectos, se proporciona un ejemplo (Palm y Nyström, 2009). En la Tabla 1 se presenta el problema junto con su versión más auténtica.

\begin{tabular}{|c|c|}
\hline Tarea & Versión más autentica \\
\hline $\begin{array}{l}360 \text { estudiantes deberán ir en } \\
\text { autobús en un viaje escolar. Cada } \\
\text { autobús tiene capacidad para } 48 \\
\text { estudiantes. ¿Cuántos autobuses se } \\
\text { necesitan? }\end{array}$ & 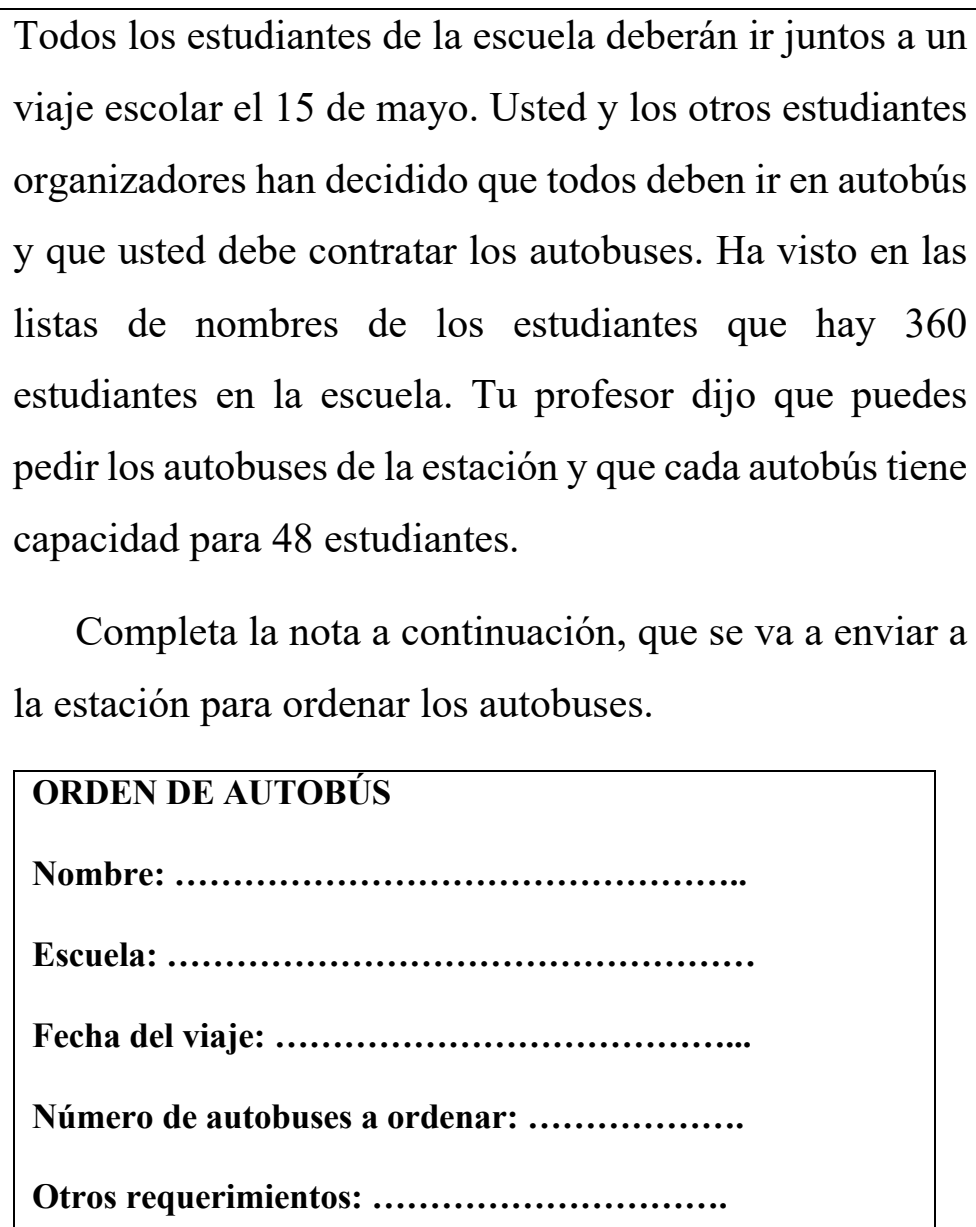 \\
\hline
\end{tabular}

Tabla 1. Tarea no auténtica con su versión más auténtica (extraído de Palm y Nyström, 2009, traducción de los autores)

Investigación e Innovación en Matemática Educativa (2020) Volumen 5 https://doi.org/10.46618/iime.85 
Como se puede observar, en la versión más auténtica ya se habla de un evento que puede ocurrir en la vida real; la pregunta es una que es habitual; los datos proporcionados coinciden con los que se pueden obtener en la vida real; el propósito y los datos proporcionados son claros y específicos. Además, en esta versión más auténtica, el estudiante puede hacer uso, además de las matemáticas escolares, de su experiencia de la vida real para resolverlo.

\section{MÉTODO}

Se realizó una investigación de tipo cualitativa. Consistió en una entrevista clínica realizada a un estudiante de 17 años de sexo masculino que cursa el segundo año de preparatoria en una escuela privada ubicada en la ciudad de Puebla, México. El entrevistado muestra un gusto y habilidad por las matemáticas, es de personalidad seria y da respuestas concisas.

La tarea planteada al estudiante fue extraída del libro de texto Geometría Analítica de Ruiz (2014) y se muestra en la Figura 1.

17. Elevaciones orográficas. La tabla y la gráfica indican la altura de algunas de las mayores cumbres de México. Estas alturas están expresadas en $\mathrm{km}$ sobre el nivel del mar, considerado éste como nivel cero. La distancia a la que se halla cada elevación de la ciudad de México, situada en el origen, puede obtenerse en km sobre el eje $x$.

a) Escribe las coordenadas de cada cima.

b) ¿Qué distancia vertical existe entre las cimas más elevada y más baja de la gráfica?

c) ¿A qué distancia se halla el Pico de Orizaba de la ciudad de México?

d) ¿Es correcto decir, con base en la gráfica, que el volcán Popocatépetl está a $130 \mathrm{~km}$ de distancia del Pico de Orizaba?

Investigación e Innovación en Matemática Educativa (2020) Volumen 5

https://doi.org/10.46618/iime.85 


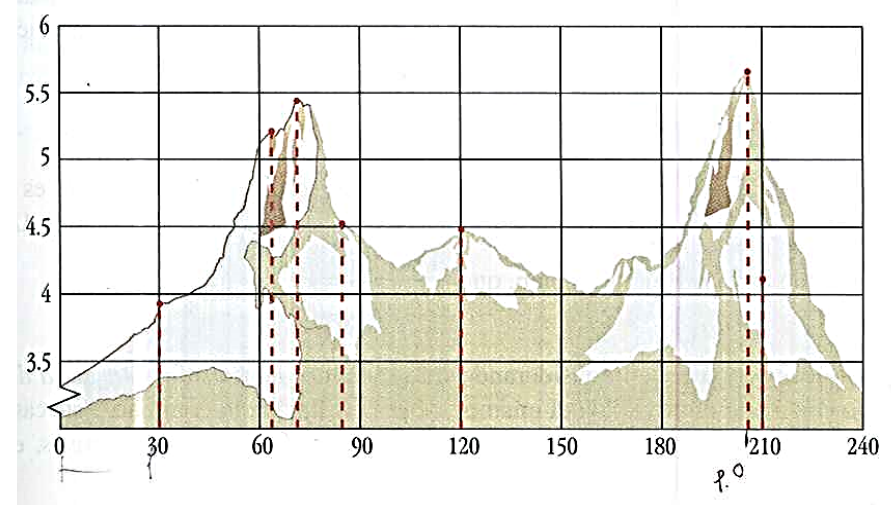

\begin{tabular}{|l|l|}
\hline \multicolumn{1}{|c|}{ Elevación } & $\begin{array}{c}\text { Altura } \\
(\mathbf{k m})\end{array}$ \\
\hline Pico de Orizaba & 5.747 \\
\hline Popocatépetl & 5.482 \\
\hline Iztaccíhuatl & 5.386 \\
\hline Nevado de Toluca & 4.558 \\
\hline La Malinche & 4.461 \\
\hline Cofre de Perote & 4.282 \\
\hline Pico del Ajusco & 3.929 \\
\hline
\end{tabular}

Figura 1. Tarea propuesta en la entrevista clínica al resolutor (Ruiz, 2014)

Las preguntas diseñadas para la entrevista se muestran en la Tabla 3. En la primera columna se muestran cada uno de los aspectos de autenticidad que se mencionan en Vicente y Manchado (2017) y en la segunda columna la guía de preguntas para ese aspecto.

\section{Aspecto}

\section{Preguntas}
1) Evento
¿Tú crees que fuera del colegio podrías encontrarte el evento que se describe? ¿Dónde?
2) Pregunta
Acerca de las preguntas, ¿tú crees que te podrían interesar fuera del colegio?
3) Datos
¿Sobre los datos que vienen en el problema, crees que los puedes obtener fuera del colegio?
4) Propósito
¿Tú crees que tiene un propósito este problema?
5) Especificidad de los datos
¿Tú crees que los objetos que aparecen en el problema están definidos?
¿Los lugares que aparecen en el problema tienen nombre propio?

Tabla 3. Guía de preguntas diseñadas para explorar la opinión del resolutor acerca de cada uno de los aspectos

Investigación e Innovación en Matemática Educativa (2020) Volumen 5

https://doi.org/10.46618/iime.85 
El procedimiento para la recolección de los datos se llevó a cabo en el semestre académico de primavera 2020. La entrevista clínica fue realizada y grabada por medio de una videoconferencia a través de la plataforma Zoom. El proceso total tuvo una duración de 59 minutos en donde primero se procedió a la resolución de la tarea por parte del estudiante, la cual tuvo una duración de 17 minutos y posteriormente se procedió a la entrevista, con una duración de 42 minutos. La tarea fue resuelta por el estudiante a la vista del entrevistador y enviada por medio de una imagen a través de WhatsApp.

Una vez que el entrevistado resolvió la tarea propuesta, se procedió a realizar la entrevista. Por medio de las preguntas, se obtuvo la opinión del resolutor acerca de si la tarea cumplía o no cada uno de los cinco aspectos. La entrevista fue transcrita inmediatamente después, considerando las expresiones corporales y lingüísticas del entrevistado y posteriormente se revisó su producción escrita para identificar las dificultades que se le presentaron en el proceso de resolución de la tarea. Como veremos en la sección de análisis, algunas se derivaron del incumplimiento de algunos aspectos de autenticidad que el mismo resolutor identificó.

Se realizó un análisis tipológico de la entrevista, como ha sido establecido en Hatch (2002), el cual consta de las nueve etapas mostradas a continuación:

i. Identificar las tipologías a analizar.

ii. Leer los datos marcando las entradas relacionadas con las tipologías o categorías establecidas.

iii. Leer la información de cada tipología registrando las ideas principales en una hoja de resumen.

iv. Buscar patrones, relaciones, temas dentro de las tipologías.

v. Leer los datos, codificar las entradas de acuerdo con los patrones identificados y mantener un registro de qué entradas corresponden a qué elementos de los patrones encontrados.

vi. Decidir si los patrones son compatibles con los datos y buscar en los datos contraejemplos de los patrones establecidos.

Investigación e Innovación en Matemática Educativa (2020) Volumen 5

https://doi.org/10.46618/iime.85 
vii. Buscar relaciones entre los patrones identificados en cada categoría o tipología.

viii. Escribir los patrones como generalizaciones de una oración.

ix. Seleccionar extractos de datos que respalden estas generalizaciones.

En nuestra investigación las tipologías que se consideraron fueron: Evento, Pregunta, Existencia de datos, Propósito, Especificidad de los datos, Estrategias de solución y Presentación. A continuación, se leyó varias veces la entrevista transcrita y se resaltaron con diferentes colores los segmentos que hacían referencia a cada tipología, se crearon archivos de cada tipología y se redactó un resumen de lo encontrado en los segmentos seleccionados de cada tipología. Se revisó si los patrones encontrados no eran contradictorios. Se buscaron patrones y temas en cada tipología, se buscaron relaciones entre los temas de las tipologías. Se hizo la selección de los segmentos de entrevista más representativos de cada tema encontrado en las diferentes tipologías.

\section{ANÁLISIS}

La entrevista clínica inició con un saludo de ambas partes y se le informó al resolutor que no importaba que sus procesos de resolución estuvieran bien o mal, pues lo que interesaba era cómo lo había hecho. A continuación, se le proporcionó la tarea y se le pidió resolverla. Una vez que terminó, se procedió a realizar la entrevista. En lo que sigue se denotará por $I$ al entrevistador y por $E$ al entrevistado.

Se procedió con la primera pregunta sobre el primer aspecto de autenticidad que es el de Evento. Se le preguntó si creía que el evento descrito en la tarea podía presentársele fuera del colegio. A continuación, se presenta el diálogo:

I: ¿Crees que el evento descrito en la tarea se te podría presentar fuera del colegio?

E: Yo creo que sí. Por ejemplo, en algún momento quiero realizar un viaje, eh... quiero saber por ejemplo cuánta distancia existe entre dos puntos, o sea,

Investigación e Innovación en Matemática Educativa (2020) Volumen 5 https://doi.org/10.46618/iime.85 
puedo saber bien cuántos kilómetros hay de diferencia o saber la variación de altura. Puedo saber otras cosas, por ejemplo, no sé, igual y si tú estás en la ciudad de México y quiero hacer, no sé, una expedición hasta la cima del pico de Orizaba pues puedo hacer una comparación y puedo saber cuántos kilómetros hay.

I: ¿Y tu opinión de si se te puede plantear fuera del colegio?

E: Ah... yo digo que sí, si se podría, en la situación de que como le comentaba se puede saber que, vaya, para ser un poco más específico de una situación en dos dimensiones, se podría dar este tipo de evento.

Como se observa, el resolutor declara que sí se puede dar este evento en la vida real; incluso proporciona varios escenarios que se le pudieran presentar. Se observa que clasifica la tarea, como un problema de distancias. Posteriormente especifica que este evento se puede dar solo si se analiza en dos dimensiones. Se observa que el resolutor no plantea alguna aplicación en la vida real de los pares coordenados, que es el otro tema que aborda la tarea. De este análisis se puede concluir que el resolutor afirma que la tarea sí cumple con este primer aspecto de autenticidad.

Continúa la entrevista, ahora sobre el segundo aspecto de autenticidad que es la Pregunta:

I: $\quad$ Y acerca de las preguntas, ¿tú crees que te podrían interesar fuera del colegio?

E: $\quad$ Ah... tal vez, porque creo que la, bueno la, las que más me interesaron, bueno serían: “¿cuál es la diferencia entre el punto más alto y el punto más bajo?” Y “cuál es la distancia de la ciudad de México al Pico de Orizaba?" O sea, esas preguntas pues vaya si interesarían, por ejemplo, si quiero hacer un viaje pues ya tengo en mente que distancia recorreré.

Investigación e Innovación en Matemática Educativa (2020) Volumen 5 https://doi.org/10.46618/iime.85 
I: $\quad$ Y, por ejemplo, ¿el inciso a)?

E: $\quad$ Ah... yo digo que no realmente, la podría llegar a utilizar, pero no se me viene a la mente ningún escenario.

I: $\quad$ Dijiste que la b) sí.

E: $\quad$ Aja, la b) y la c).

I: $\quad$ ¿Ylad)?

E: La d) pues... es un complemento ¿no? O sea, el hecho de poder afirmar si existe esa distancia, pero se necesitarían datos, o sea datos específicos y ya.

De lo anterior se observa que al resolutor sólo le interesarían, fuera del colegio, dos preguntas. En estas sólo se habla de distancias verticales y horizontales entre las cimas. Declara que el primer inciso no se lo podría formular fuera del colegio ya que no se le viene a la mente ningún escenario en dónde utilizar coordenadas. Acerca del último inciso, afirma que faltarían datos para hacer esa pregunta. Entonces, se puede afirmar que según la perspectiva del resolutor no se cumple con el segundo aspecto de autenticidad, la Pregunta.

Siguiendo con el tercer aspecto se le hace la pregunta:

I: $\quad$ Y sobre los datos que vienen en el problema, ¿crees que los puedes obtener fuera del colegio?

E: $\quad$ Los datos, eh... sí. Puedo hacer, podría usar otros métodos como eh, eh hacer las expediciones, o si no utilizar un tacómetro para medir las distancias, guiarme a través de mapas y otros, o para las alturas pues igual podría a través de una carta topográfica podría ver, vaya a qué altura se encuentra.

El resolutor afirma que los datos que se presentan en la tarea sí se pueden obtener en la vida real, incluso da una variedad de formas. Posteriormente, dialogando con el entrevistador logra dar otras formas de conseguir información, como el buscar en un libro o lo más común, buscar en

Investigación e Innovación en Matemática Educativa (2020) Volumen 5 https://doi.org/10.46618/iime.85 
internet. De lo anterior se puede afirmar que desde la perspectiva del estudiante sí se cumple con el tercer aspecto de autenticidad, los Datos.

Continúa la entrevista con el cuarto aspecto:

I: ¿Tu crees que tiene un propósito el ejercicio?

E: $\quad$ Mmmm sí, yo creo que te enseña bien a medir las distancias, tanto verticales como horizontales y pues más complejamente te ayuda a medir las distancias diagonales

I: Ok. ¿El propósito es medir?

E: $\quad$ Mju

I: ¿Medir qué?

E: $\quad$ Distancias de puntos.

I: ¿Y qué tema crees que está abarcando especificamente?

E: Geometría analítica porque mide la distancia, por ejemplo, en la pregunta b) del punto más bajo que dan en la tabla al punto más alto, entonces ya se hace una. Se genera un dato que representa la diferencia entre el punto más bajo y el punto más alto.

De lo anterior se puede observar que el resolutor sí detecta un propósito, que es el de medir distancias, verticales, horizontales y diagonales, aunque comete un error porque en el problema no se pide medir distancias diagonales. Posteriormente, afirma que se trata de un tema de geometría analítica, pero no menciona que se trata del tema de coordenadas cartesianas, las cuales se piden en el primer inciso. Nótese que una vez que se pide se ubiquen las coordenadas cartesianas de los puntos correspondientes a las cimas de las cumbres en el inciso a), no se utilizan en los incisos siguientes, es decir, el uso de las coordenadas cartesianas no tiene un propósito claro en la tarea.

Investigación e Innovación en Matemática Educativa (2020) Volumen 5 https://doi.org/10.46618/iime.85 
Debido a ello, desde la perspectiva de los investigadores, la tarea no cumple el cuarto aspecto de autenticidad referente al Propósito de la tarea. Sin embargo, desde el punto de del resolutor, sí se cumple.

Continuando la entrevista, con el quinto aspecto que tiene que ver con la Especificidad de los datos, se le cuestionó:

I: ¿Y tu crees que los objetos que aparecen en el problema están definidos?

E: No del todo bien.

I: ¿Por qué no del todo bien?

E: Porque sí tuve un poco de problemas, o sea porque solo venía la gráfica vertical y un puntito, pero no me representaba nada, o sea no podía ver alguna montaña en especifico.

I: ¿Entonces lo que no estaría definido serían las montañas o qué?

E: Es que nada más vi dos o tres, pero si por ejemplo hubieran puesto una figura por cada punto igual sería más comprensible.

El resolutor afirma que los lugares no están bien definidos. Uno de los motivos que plantea es que en la gráfica no se pueden identificar todas las montañas y que el dibujo genera confusión.

Asimismo, cuando se le pregunta su opinión general acerca de la tarea, externa problemas relacionados con este aspecto:

I: $\quad$ Bueno, ahora quisiera saber tu opinión en general acerca de este problema.

E: Pues yo digo que está bien, sí es comprensible, solo que, sí tuve muchas dudas al momento de encontrar las distancias sobre el eje $x$, porque sólo ponía distancias de 30 en 30, pero marcaba puntos a la mitad, entonces yo digo que no especifica muy bien. Por ejemplo, si ve, puse muchas aproximaciones y no sé si realmente fueron esas distancias. Puede ser que

Investigación e Innovación en Matemática Educativa (2020) Volumen 5 https://doi.org/10.46618/iime.85 
hayan sido diez o dos puntos más entonces sí como que falta una mayor definición sobre el eje.

Como se observa, otro de los motivos por el cual el entrevistado afirma que los lugares no están bien definidos es porque no son claras las distancias sobre el eje $x$. Esta situación obliga al estudiante a realizar cálculos aproximados, casi imposibilitándolo a llegar al resultado planteado en el libro de texto.

A continuación, nuevamente se le interroga acerca de su comprensión en la especificidad de los datos:

I: Bueno. ¿En el ejercicio comprendes todos los datos que vienen en la tabla y en la gráfica?

E: Ah, en la tabla sí, si comprendo, pero en la gráfica no porque ah ... porque aparte no, ¿cómo se dice?, le comentaba que no venían puntos tan específicos para saber la distancia entre los puntos intermedios, este, no encuentro congruencia entre las medidas del eje $x y$ del eje $y$, porque abajo en el eje $x$ venían en cantidades de cien, bueno de centenas y en el eje y solo venían cantidades de unidades y puntos, entonces yo digo que sería mejor para una mayor precisión sería nivelar las escalas.

En su repuesta el resolutor menciona que no hay congruencia entre las medidas del eje $x \mathrm{y}$ del eje $y$. Nótese la contradicción en la tarea en que, por un lado, piden resultados exactos en el libro de texto hasta el orden de centésimas en las alturas verticales, y por otro lado, los datos son inespecíficos en la gráfica que proporciona. Esto provocó que los datos de las distancias horizontales proporcionados por el resolutor fueran aproximados.

Se continuó explorando este aspecto de autenticidad con el estudiante:

I: Y todos los lugares que mencionan tienen un nombre, ¿cierto?

E: Sí, pues sí.

Investigación e Innovación en Matemática Educativa (2020) Volumen 5 https://doi.org/10.46618/iime.85 
Sobre si los lugares tienen nombre propio afirma que sí, que tanto los volcanes como la ciudad que menciona lo tienen.

De todo el análisis de este aspecto de la Especificidad de los datos se puede concluir que, desde la perspectiva del resolutor, aunque los lugares tienen nombre propio, no están bien definidos. Por lo tanto, la tarea no cumple el quinto y último aspecto.

En síntesis, desde la perspectiva del resolutor la tarea no es auténtica porque no cumple con los aspectos de Pregunta y Especificidad de los datos.

Una vez que se analizaron los cinco aspectos de autenticidad, se procedió a hacer preguntas sobre sus procesos de resolución de la tarea. Inicialmente se le pregunta acerca de que si la tarea se le presentara en la vida real la resolvería exactamente igual:

I: Pero ¿y los procedimientos de resolución que harías, serían de la misma forma?

E: Ah, yo digo que sí.

I: ¿Lo resolverías exactamente igual?

E: Yo digo que sí.

Aquí afirma que sí la resolvería exactamente igual que en la vida real. Aunque en este trabajo no estamos analizando todos los aspectos propuestos por Palm (2006) respecto a una tarea auténtica, se observa que esta afirmación coincide con el sub-aspecto disponibilidad, que es parte del aspecto Estrategias de solución. Este sub-aspecto se refiere a que debe haber coincidencia entre las estrategias de solución disponibles para los estudiantes que resuelven la tarea escolar y las estrategias disponibles para las personas correspondientes en la vida real. Como se observa en el fragmento de entrevista, el resolutor opina que sí lo cumple desde su perspectiva.

Continuando con la resolución del problema, se le cuestiona sobre su respuesta al inciso a) de la tarea

I: ¿Cómo obtuviste las coordenadas de la cima 3: $(62,5.38) ?$

Investigación e Innovación en Matemática Educativa (2020) Volumen 5 https://doi.org/10.46618/iime.85 
E: El 5.38 es del eje y y lo obtuve de la tabla y el 62 fue un aproximado

I: $\quad$ Es el Iztaccíhuatl, ¿cierto?

E: $\quad$ Ajá

I: $\quad$ 62, ¿cómo hiciste la aproximación?

E: Pues dividí del 60 al 90 porque de ahí yo tenía una pequeña referencia que era la tercera parte y la tomé como 10, o sea, y ya lo dividí y más o menos me salió como 62, 63 igual. Entonces fue una aproximación.

I: $\quad$ Ok. Por eso elegiste el 62, te pareció que más o menos ahí estaba el 62. ¿Así hiciste con las demás?

E: Este, sí, más o menos.

El resolutor declara que como los datos en el eje $x$ no son específicos tuvo que hacer una aproximación de su respuesta, dando las coordenadas $(62,5.38)$ como se observa en la Figura 2. Cabe mencionar que el libro proporciona la respuesta $(65,5.38)$. De aquí se puede concluir que, al no cumplir el aspecto de especificidad de los datos, el alumno no puede llegar a la respuesta esperada.

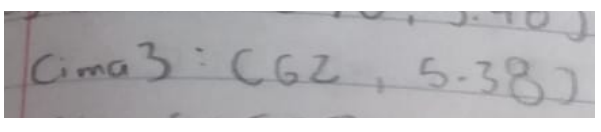

Figura 2. Respuesta del inciso a) proporcionada por el resolutor.

Aquí se observa una dificultad en el estudiante al no proporcionar la respuesta que solicita el libro y la relación que existe entre este hecho y el incumplimiento del aspecto de Especificidad de los datos. La falta de este aspecto genera que el estudiante no pueda dar la respuesta proporcionada en el libro, no porque tenga problemas en sus procesos de resolución, sino porque la tarea planteada no es auténtica.

De igual forma sucede en las preguntas b) y c) de la tarea, el estudiante tuvo que dar respuestas aproximadas. En el inciso b) afortunadamente sí coincidió con la proporcionada en el libro. A continuación, se muestra el fragmento de la entrevista donde se le cuestiona sobre estas.

Investigación e Innovación en Matemática Educativa (2020) Volumen 5 https://doi.org/10.46618/iime.85 
I: $\quad$ La siguiente pregunta dice: “ ¿A qué distancia se haya el Pico de Orizaba de la Ciudad de México?” ¿Cómo la obtuviste?

E: Eh, igual por aproximación porque... ah...pues vaya, como no tienen un dato conciso tomé como referencia el 210 e igual por aproximación dije que había una diferencia de $5 \mathrm{~km}$ aproximadamente.

I: La siguiente pregunta dice: “¿Es correcto decir con base en la gráfica que el volcán Popocatépetl está a 130 km de distancia del Pico de Orizaba? ” Y tú me pones que sí. ¿Cómo lo resolviste?

E: Pues sí y no, porque como le digo fue un estimado y pues la diferencia real da de 135 , entonces pues entra dentro del 130.

La respuesta que el resolutor proporciona al inciso c) es $135 \mathrm{~km}$, puesto que está trabajando con datos aproximados. Afirma que la respuesta es correcta pues 135 se aproxima a 130.

Nuevamente la respuesta incorrecta del estudiante y la ambigüedad al decir que lo resolvió "sí y no" se debe a la falta de especificidad en los datos. No es que el estudiante tenga problemas en sus procesos de comprensión del problema y de resolución, sino en la falta de cumplimiento del aspecto Especificidad de los datos. Un profesor que evalúe solo la respuesta del problema, según el libro de texto, diría que el estudiante resolvió incorrectamente el problema, cometiendo un error.

Se continúa con el inciso d) del problema: ¿Es cierto decir con base en la gráfica que el volcán Popocatépetl está a 130 km de distancia del Pico de Orizaba?

I: Bueno la pregunta nos dice: “¿Es correcto decir con base en la gráfica que el volcán Popocatépetl está a $130 \mathrm{~km}$ de distancia del Pico de Orizaba?”. ¿Tú crees que es correcto?

E: Sí.

I: (Se le muestra la figura del problema) Esto lo que me dice es la distancia que hay de la Ciudad de México a cualquier volcán, entonces yo quiero saber cuál

Investigación e Innovación en Matemática Educativa (2020) Volumen 5 https://doi.org/10.46618/iime.85 
es la distancia del Pico de Orizaba al volcán Popocatépetl, ¿esta gráfica me sirve para resolverlo?

E: iAh! Ah no, pues porque me da las distancias a la Ciudad de México.

I: Entonces, ¿esta gráfica me sirve?

E: No.

El resolutor afirma que la gráfica no es suficiente para dar una respuesta a la pregunta del inciso d) del problema.

Nuevamente, se le pregunta sobre si la información presentada en la gráfica es útil para contestar la pregunta:

I: $\quad$ ¿Me sirve la gráfica para saber cuál es la distancia entre el Pico de Orizabay el Popocatépetl?

E: No.

I: Entonces, ¿Cuál sería tu respuesta a la última pregunta? (inciso d) del problema)

E: No.

I: $\quad$ Y acerca de los volcanes que los presenta como en una línea, ¿qué opinas?

E: Considero que está bien porque representa la distancia lineal entre la Ciudad de México y cada uno de los volcanes.

I: ¿Qué es lo que te hace pensar la gráfica, así como se muestra?

E: Pues que marca distancias desde el origen a cada uno de los puntos del plano cartesiano

I: ¿Y cómo parece que están los volcanes?

E: Conectados en una línea recta.

Investigación e Innovación en Matemática Educativa (2020) Volumen 5 https://doi.org/10.46618/iime.85 
Derivado de la imagen que aparece en la gráfica (Figura 3), pareciera ser que todos los volcanes y la ciudad de México (en el origen) se encuentran alineados, más aún, parece en la gráfica que algunos volcanes son parte de otros. Esto provoca confusión en el momento de la resolución y durante la entrevista, nuevamente derivado de la falta de especificidad de los datos.

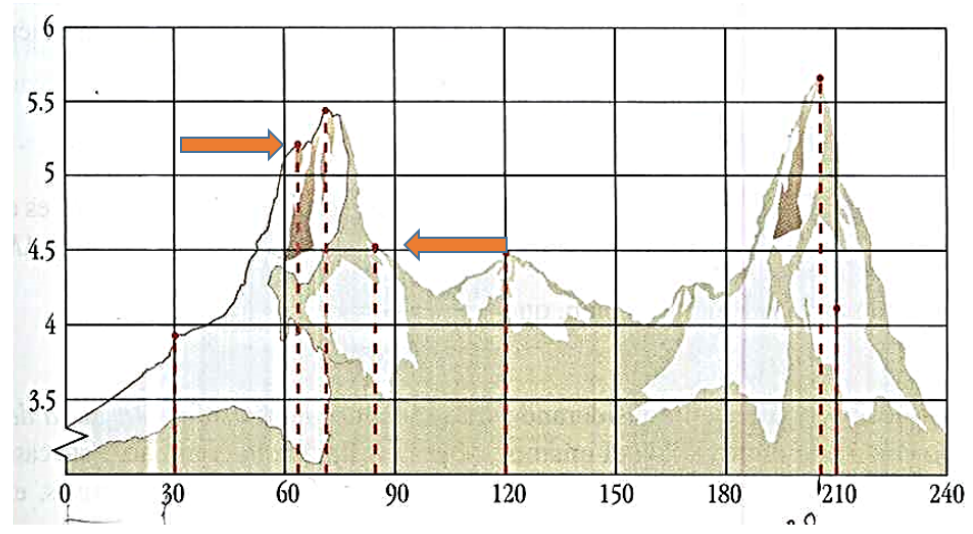

Figura 3. Errores en la representación gráfica de los volcanes.

Por último, se le pregunta:

I: ¿Aparte de la gráfica, todo estuvo bien?

E: Sí. Sólo que, si la segunda idea del planteamiento del problema se pudiera simplificar, o vaya, de hacerlo de una manera más explícita sería mucho mejor.

I: ¿De cuál pregunta, perdón?

E: De la, del planteamiento de la situación, la segunda idea que dice: de la Ciudad de México sobre el eje x no recuerdo bien. Pero la segunda idea, yo digo que sí se podría simplificar, para que quede un poco más claro.

I: ¿Dónde dice: "la distancia a la que se halla cada elevación a la ciudad de México está situada en el origen, puedo obtenerse en km", a eso te refieres?

E: Sí. Yo lo comprendí como que el eje x era la distancia que había hasta los puntos, hasta las montañas y los volcanes. 
I: $\quad$ Efectivamente a eso se refiere. ¿Crees que podría estar más simplificado o mejor redactado?

E: Eh sí, yo creo que se podría hacer más simple en la cuestión de que se podría decir que las distancias señaladas en el eje x se refiere a la distancia entre la Ciudad de México que está en el origen hasta los volcanes, los puntos.

I: $\quad$ Para que sea más claro.

E: Vaya sí.

El entrevistado saca a relucir otro de los sub-aspectos considerados en Palm (2006), El uso del lenguaje, del aspecto Presentación que se refiere a la terminología, la estructura de la oración y la cantidad de texto que hay en la tarea escolar. Aquí se da a notar que el incumplimiento de este aspecto afectó en los procesos de resolución y el uso de las matemáticas.

\section{CONCLUSIONES}

El objetivo de esta investigación fue identificar cuáles son los aspectos de una tarea propuesta que percibe como auténticos un estudiante de bachillerato y cómo se relacionan con dificultades que presenta en su resolución. Con base en los resultados obtenidos en este estudio de caso derivado de la entrevista clínica y su análisis, podemos concluir que el resolutor califica la tarea propuesta como no auténtica, ya que no cumple con dos de los aspectos de autenticidad considerados por Palm y Nyström (2009). Desde su perspectiva, no cumple con la Pregunta y la Especificidad de los datos.

Con respecto al aspecto de Evento, el estudiante dice que sí se puede presentar la situación del problema en la vida real porque interpreta que se trata de un problema de distancias, más que como un problema de pares ordenados pues solo la primera pregunta tenía que ver con este tema. Su respuesta también está vinculada a los aspectos Propósito y Datos, pues el estudiante afirma que sí se pueden presentar en la vida real. También, como resultado de la entrevista, se pudo concluir

Investigación e Innovación en Matemática Educativa (2020) Volumen 5 https://doi.org/10.46618/iime.85 
que desde la perspectiva del resolutor la tarea tampoco cumple con dos aspectos más: Estrategias de solución y Presentación, descritos en la propuesta general de Palm (2009).

Con respecto a la relación entre los aspectos de autenticidad y su relación con los procesos de resolución del resolutor se puede concluir que la gráfica del problema no mostraba valores exactos en las ubicaciones de los volcanes, sin embargo, solicitaba una respuesta exacta en el libro de texto, lo cual evidentemente el resolutor no pudo cumplir a consecuencia de la falta de autenticidad en los Datos.

Asimismo, la gráfica muestra a tres volcanes en una sola montaña y en una línea recta junto con la Ciudad de México, lo cual no sucede en la vida real. Esto provocó que el resolutor se confundiera al momento de calcular distancias y no pudiera responder la pregunta d) del problema.

En síntesis, se pudo observar que el estudiante tenía claros los procedimientos matemáticos a realizar del cálculo de pares ordenados y distancias horizontales y verticales, pero no pudo completar la tarea por fallas en la autenticidad de la tarea. Los más notorios se dan cuando utiliza la gráfica de los volcanes.

Se puede sugerir que el docente analice previamente los problemas a implementar con sus estudiantes desde los aspectos de autenticidad de Palm y Nyström (2009). Esto puede evitar que los alumnos se confundan al momento de su resolución ya que existen problemas que pretenden tener un contexto pero que no logran reflejar la realidad completamente, causando dificultades; es decir, les falta autenticidad.

De este trabajo de investigación se puede concluir que analizar la autenticidad de una tarea desde la perspectiva de un buen resolutor puede proporcionar información al docente acerca de qué aspectos pueden mejorarse en la tarea planteada, repercutiendo en mejores actividades para proponer a los estudiantes. Incluso podría pedirse a los estudiantes analizar la autenticidad de tareas propuestas por el docente o tareas de libros de texto y proponerles realizar un rediseño para hacerlas más auténticas y de esta manera coadyuvar en una mayor comprensión de conceptos y métodos de resolución.

Investigación e Innovación en Matemática Educativa (2020) Volumen 5 https://doi.org/10.46618/iime.85 


\section{REFERENCIAS}

Aiken, L. R. (1980). Content validity and reliability of single items or questionnaires. Educational and Psychological Measurement, 40(4), 955-959. https://doi.org/10.1177/001316448004000419

Aiken, L. R. (1985). Three coefficients for analyzing the reliability and validity of ratings. Educational and Psychological Measurement, 45(1), 131-142. https://doi.org/10.1177/0013164485451012

Becker, J. P., y Shimada, S. (1997). The open-ended approach: A new proposal for teaching mathematics. Reston, Virginia: National Council of Teachers of Mathematics.

Blum, W. y Niss, M. (2014). Applied Mathematical Problem Solving, Modelling, Applications, and Links to Other Subjects: State, Trends and Issues in Mathematics Instruction Author(s): Werner Blum and Mogens Niss Source: Educational Studies in Mathematics, 22(1), 3768.

Boaler, J. (1994). When Do Girls Prefer Football to Fashion? An analysis of female underachievement in relation to 'realistic' mathematic contexts. British Educational Research Journal, 20(5), 551-564. https://doi.org/10.1080/0141192940200504

Codina, A., Rivera, A. (2001). Hacia una instrucción basada en la resolución de problemas: los términos problema, solución y resolución. En P. Gómez y L.R. Romero (Eds.). Iniciación a la investigación en didáctica de la matemática: homenaje al profesor Mauricio Castro. Granada, España: Universidad de Granada

De León, W. (2020). La autenticidad de los problemas matemáticos en contextos de temperatura en los libros de texto de México y Colombia. (Tesis de maestría no publicada). Benemérita Universidad Autónoma de Puebla, México.

Duschl, R. A. y Gitomer, D. H. (1996). Project Sepia Design Principles. Paper presented at the anual meeting of American Educational Research Association, New York.

Investigación e Innovación en Matemática Educativa (2020) Volumen 5

https://doi.org/10.46618/iime.85 
Guerrero, A. C., Carrillo, J., Contreras, L. C. (2014). Problemas de sistemas de ecuaciones lineales en libros de texto de $3^{\circ}$ ESO. En M. T. González, M. Codes, D. Arnau y T. Ortega (Eds.), Investigación en Educación Matemática XVIII (pp. 395-404). Salamanca: SEIEM.

Hatch, J. (2002). Doing qualitative research in education setting. New York, NY: State University of New York Press. https://doi.org/10.1155/2013/704806

Jimenez , M. P. (1998). Diseño curricular: indagación y razonamiento con el lenguaje de las ciencias. Enseñanza de las Ciencias, 16(2), pp. 203-316.

Labarrere, A. (1996). Inteligencia y Creatividad en la escuela, Revista Educación (88), pp. 20-25.

Leont'ev, A. (1975). Dieyatelinocti, soznaine, i lichynosti [Activity, consciousness, and personality]. Moskva: Politizdat

Martínez, G. (2019). La cubicación de madera como un problema geométrico del contexto real diseñado e implementado en un bachillerato comunitario. Tesis de maestría no publicada. Benemérita Universidad Autónoma de Puebla, México.

Mason, J. y Johnston-Wilder, S. (2006). Designing and using mathematical tasks. York, UK: QED Press.

Palm, T. (2006). Word Problems as Simulations of Real-World Situations: A Proposed Framework. Learning of Mathematics, 26(1), 42-47.

Palm, T. (2008). Impact of authenticity on sense making in word problem solving. Educational Studies in Mathematics, 67(1), 37-58. https://doi.org/10.1007/s10649-007-9083-3

Palm, T., y Nyström, P. (2009). Gender Aspects of Sense Making in Word Problem Solving. Journal of Mathematical Modelling and Application, 1(1), 59-76.

Penfield, R. D., y Giacobbi, P. R. (2004). Applying a score confidence interval to Aiken's item content-relevance index. Measurement in Physical Education and Exercise Science, 8(4), 213-225. https://doi.org/10.1207/s15327841mpee0804_3

Ruiz, J, (2014). Geometría analítica, Cuidad de México, México. Patria.

Investigación e Innovación en Matemática Educativa (2020) Volumen 5 https://doi.org/10.46618/iime.85 
Vicente, S., y Manchado, E. (2017). Dominios de contenido y autenticidad: un análisis de los problemas aritméticos verbales incluidos en los libros de texto españoles. PNA, 11(2017), 253-279.

Watson, A., Ohtani, M., Ainley, J., Bolite, F. J., Doorman, M., Kieran, C., Leung, A. (2014). Introduction. En C. Margolinas (Ed.). Task Design in Mathematics Education (pp. 7-13). Proceedings of ICMI Study 22. Oxford, United Kingdom.

Investigación e Innovación en Matemática Educativa (2020) Volumen 5 https://doi.org/10.46618/iime.85 